\title{
Cartografías de desigualdades en los cuidados en clave de Trabajo Social
}

\section{Lorena Guerriera}

Licenciada en Trabajo Social (Universidad Nacional de Entre Ríos)

Correo: lorenaguerriera@gmail.com

\section{Carina Carmody}

Licenciada en Trabajo Social (Universidad Nacional de Entre Ríos)

Magister en Trabajo Social (Universidad Nacional de Entre Ríos)

Correo: ccarmody@fts.uner.edu.ar

\section{Alicia Genolet}

Licenciada en Servicio Social (Universidad Nacional de Entre Ríos)

Magister en Metodología de Investigación

Científica (Universidad Nacional de Entre Ríos)

Correo: asgenolet@gmail.com

\section{Nadia Ahumada}

Licenciada en Trabajo Social (Universidad Nacional de Entre Ríos)

Correo: nadia.eahumada@gmail.com 
Resumen

Este trabajo retoma aspectos trabajados en el proyecto de investigación "Prácticas de cuidado de niños menores de 4 años que realizan las familias en situación de pobreza en la zona noroeste de la ciudad de Paraná. Un estudio desde la perspectiva de género" (2015-2018)

El objetivo de este artículo es compartir algunas reflexiones con relación a las herramientas y metodologías utilizadas. En un primer momento nos enfocamos en el uso de cartografías recuperando los trabajos de las pioneras feministas del Trabajo Social estadounidense de principios de siglo XX. En la segunda parte trabajaremos con las cartografías elaboradas en el estudio realizado por el equipo de investigación. A partir de un conjunto de estadísticas públicas realizamos un mapeo que da cuenta de la distribución geográfica de la oferta de cuidados infantiles. Finalmente, la propuesta del trabajo brinda herramientas para comprender, representar y mostrar cuales son los modos en que se expresan los patrones de desigualdad de los cuidados infantiles vinculados a las demás desigualdades en términos sociales, económicos y de género.

\section{Palabras claves}

cartografías, cuidados, género
Abstract

This article takes up aspects worked on in the research project "Childcare practices under 4 years old, carried out by families in poverty in the northwestern part of the city of Paraná. A study from a gender perspective" (2015-2018) The aim of this article is to share some reflections about the tools and methodologies used in the research. At first, we focus on the use of cartographies recovering the work of feminist pioneers of American social work of the early twentieth century. In the second part we will work with the cartographies elaborated in the study carried out by the research team. From a set of public statistics, we carry out a mapping that accounts for the geographical distribution of the child care offer. Finally, the work proposal provides tools to understand, represent and show what are the ways in which the patterns of inequality of child care linked to other inequalities in social, economic and gender terms are expressed.

Keywords

cartographie, care, gender 


\section{Introducción}

Este artículo retoma aspectos trabajados en el proyecto de investigación que se desarrolló en la Facultad de Trabajo Social de la Universidad Nacional de Entre Ríos, denominado "Prácticas de cuidado de niños menores de 4 años que realizan las familias en situación de pobreza en la zona noroeste de la ciudad de Paraná. Un estudio desde la perspectiva de género". En esta investigación utilizamos las cartografías, entendidas como una herramienta, para graficar y visibilizar la distribución de las desigualdades.

Nos proponemos compartir en este artículo algunas reflexiones con relación a las herramientas y metodologías utilizadas: El uso de cartografías recuperando los trabajos de las pioneras feministas del Trabajo Social estadounidense de principios de siglo XX. En la segunda parte exponemos algunos resultados de la utilización de las cartografías en la investigación.

\section{Las pioneras feministas del Trabajo Social y el uso de cartografías}

Desde el campo de la historiografía desde hace varias décadas se han venido denunciando la exclusión de las experiencias de las mujeres en el relato de la historia en general. Como trabajadoras sociales, siguiendo un movimiento impulsado por autoras como Bibiana Travi y el Grupo de investigadores/as en Trabajo Social (GIITS), colocamos la mirada en el pasado de la disciplina y el foco en las experiencias de las pioneras de Trabajo Social desde una perspectiva que supone narrar desde un modo renovado la historia profesional.

Asimismo, señalamos y celebramos la expansión de estudios vinculados a la historia del Trabajo Social, en particular en el contexto argentino, señalando la importancia de recuperar los procesos locales desde una perspectiva plural y heterogénea de la historia de la disciplina.

En la bibliografía referida al surgimiento del trabajo social estadounidense, aparecen perfilándose en lo que fueron las últimas décadas del siglo XIX y principios del XX, dos tendencias: La representada 
por las sociedades de organización de la caridad (C.O.S.) y los denominados "Settlement House Movement".

La primera, más partidaria de la intervención individualizada y familiar, y más conocida por los trabajadores sociales latinoamericanos por los textos de historia del trabajo social a partir de la figura de Mary Richmond. La segunda, denominada movimiento de los settlements, no tan difundida en los libros de historia del trabajo social, estuvo representada por Jane Adams y las primeras militantes de la igualdad racial, del feminismo y pacifistas.

Los relatos acerca de la historia de trabajo social en América Latina se enfocaron en la figura de Mary Richmond y la organización de la COS) aunque desde una mirada desvalorizante que descalifica y ridiculiza su legado por considerarlo conservador, tradicional y funcionalista.

El movimiento de los settlements, la figura de Jane Adams y de otras pioneras del trabajo social, solo ha salido a la luz a partir de los estudios recientes. La perspectiva desde la cual se narró esa historia disciplinar podría resumirse en lo que Bibiana Travi (2015) menciona como "epistemicidio" que, haciendo un uso amplio del concepto elaborado por Boaventura de Sousa Santos, define como un proceso sistemático de negación, silenciamiento y desvalorización de nuestro acervo de conocimientos y experiencias profesionales.

Si bien no es el propósito de este trabajo detenernos particularmente en la historia del trabajo social o en los relatos hegemónicos acerca de la historia en la disciplina, nos proponemos recuperar en esta oportunidad el uso de ciertos desarrollos metodológicos y la producción de conocimientos que las pioneras de trabajo social han desarrollado.

Estas pioneras norteamericanas nacidas a mediados del siglo XIX, al igual que otras mujeres de clase media y alta de la época, comenzaron a comienzos del siglo XX a luchar para tener acceso a la educación en las universidades. Esta incorporación de las mujeres a la educación superior, tal como señala Travi (2015), se realiza no exenta de un debate que, con argumentos patriarcales y supuestamente científicos, señalaba la inferioridad e incapacidad mental de las mujeres, 
las consecuencias de la educación universitaria para la salud reproductiva de las jóvenes y los efectos en la disminución de los índices de fecundidad.

La incorporación de las mujeres al ámbito universitario sin duda estuvo asociada a los desarrollos de la disciplina e institucionalización del trabajo social. Jane Adams, que tal como mencionamos fundó el movimiento de los setllements, fue una destacada investigadora y académica en el Departamento de Sociología de la Universidad de Chicago. En 1931 esta pionera del trabajo social es galardonada con el Premio Nobel de la Paz. Addams se hizo célebre durante el gran conflicto bélico de principios de siglo por su labor pacifista y por su militancia a favor del voto femenino.

A lo largo de su vida creó y presidió numerosas instituciones. En 1915 contribuyó a formar la Liga Internacional de las Mujeres para la Paz y la Libertad y en 1920, junto a líderes socialistas fundaron la American Civil Liberties Union y la National Association for the Advancement of Colored People (NAACP). Fue presidenta de la National Amercian Woman Suffrage Association, miembro de la Liga Antiimperialista Americana y de la American Sociology Association.

En 1889 funda la Hull House el primer setllement de EEUU. Las actividades de estos centros barriales eran múltiples: Servicios de salud, de ayuda social, jardines de infantes, clases, charlas y conferencias de temas tanto históricos como políticos, artísticos, en los que contaban con la presencia permanente de artistas, militantes, universitarios, y en particular los máximos referentes del pragmatismo filosófico y el interaccionismo simbólico como John Dewey, George Mead del departamento de sociología de la Universidad de Chicago.

Las residentes del settlement de Chicago o Hull House, eran casi todas mujeres con formación profesional de grado y posgrado, y este hecho contribuyó a que estos centros se fueran convirtiendo en núcleos de investigación social aplicada, orientada a producir conocimientos que permitieran fundamentar la necesidad de reformas legislativas y políticas sociales. Los estudios realizados fueron el fundamento de la promulgación de la primera ley del menor y de la jornada de 8 horas para las mujeres, de la ley de inspección de las condiciones de trabajo 
en las fábricas y de las indemnizaciones laborales. Se trataba de una institución en la que la investigación social constituía una seña de identidad. Uno de los libros más difundidos de Jane Adams fue "Hull House, Maps and Papers" de 1895 que constituye un hito de la investigación en Trabajo Social y de la sociología norteamericana. En este libro se despliegan técnicas de investigación novedosas para la época como es la localización en un mapa de cada uno de los problemas o variables demográficas de estudio de la ciudad. En esos planos la pobreza, ingresos, trabajo, inmigración y condiciones de vida se representan territorialmente con el objetivo de fundamentar la necesidad de políticas públicas.

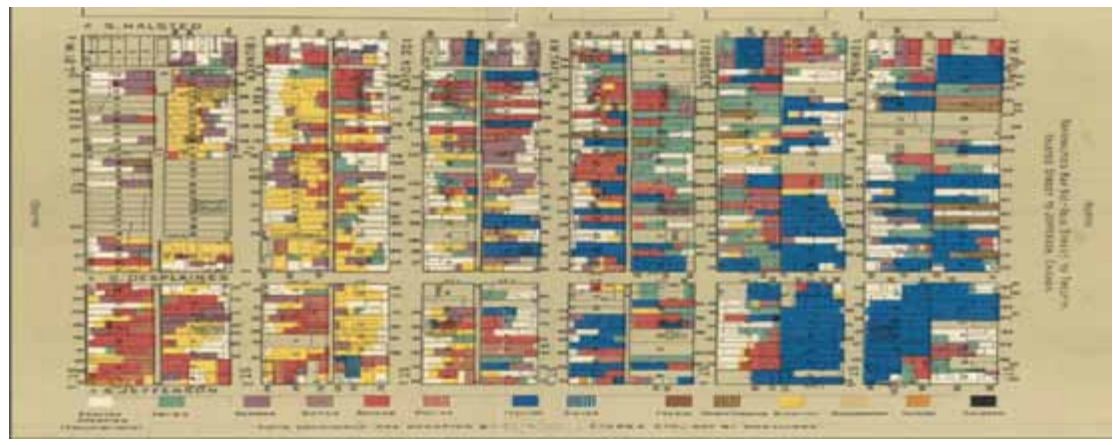

Fuente:

https://publications.newberry.org/makebigplans/plan_images/greeley-nationalities-map-no-2-1895 (Acceso agosto 2019)

Con respecto al trabajo de campo sociológico, los historiadores de métodos sostienen que fue la Escuela de Chicago, fuertemente asociada a su concepción y práctica de investigación empírica en torno de los fenómenos urbanos, la pionera de la investigación cualitativa. Estos autores destacan el rol innovador en el desarrollo de un estilo de investigación, la generación de nuevas estrategias para la recolección de datos y su énfasis en la producción de información de primera mano (Taylor y Bogdan, 1986; Denzin y Lincoln, 1994; Vasilachis, 1993).

Roxana Guber (2002) señala que prácticamente ninguno de estos trabajos de la escuela de Chicago se había basado en datos obtenidos 
por los autores, ese material empírico había sido recolectado por asistentes sociales provenientes de instituciones vinculadas a la reforma settlements).

Teresa Matus (2017) señala que las cartografías y mapas sociales han sido relevantes en el surgimiento de Trabajo social. "Sin embargo, por la forma en que hegemónicamente en nuestras escuelas se narran los orígenes de la disciplina, los aportes de esas mujeres pasan completamente desapercibidos y son colocados en el marco de subordinación” (2017:139).

La autora traza un recorrido por alguna de estas investigaciones señalando la importancia de la relación entre los mapas y los relatos y la necesidad de considerar la construcción de cartografías como una agenda pendiente de trabajo social

\section{Cartografías acerca de los cuidados en clave de género}

La premisa de la que parte esta investigación denominada "Prácticas de cuidado de niños menores de 4 años que realizan las familias en situación de pobreza en la zona noroeste de la ciudad de Paraná. Un estudio desde la perspectiva de género" es la siguiente: los diferentes grupos sociales resuelven las tensiones entre el trabajo y familia, y en general las tareas vinculadas al cuidado de niños y niñas, haciendo uso de diferentes estrategias y recursos (servicios e infraestructura estatal, de redes familiares, comunitarias, etc.). Una política estatal ausente o insuficiente en tema de cuidados, reproduce o genera la mercantilización o la familiarización de esta necesidad (siendo asumida principalmente por alguna mujer del grupo familiar), configurando una lógica particular en los modos de organizar socialmente su provisión. El estudio de la organización social de los cuidados posibilita dar cuenta del modo particular que asume el sistema de protección de derechos, y en este caso, el derecho al cuidado (Pérez Orozco, 2009). Siguiendo a la mencionada autora, la posibilidad -o no- de recibir cuidados es un indicador de desigualdad social, marcada por la accesibilidad, calidad y cobertura de redes (familiares, comunitarias, institucionales). Asimismo, la ausencia de una infraestructura y políticas de cuidado públicas tiene consecuencias en los 
tiempos y cargas de la población femenina, sobre todo en situación de pobreza, y reproduce o consolida las desigualdades vinculadas a lo económico, social y de género.

En la primera etapa de la investigación, estudiamos el acceso y disposición de la provisión de cuidados o infraestructura de servicios públicos, comunitarios y privados, por medio del uso de cartografías o mapas de cuidados en la zona de referencia de la investigación.

En un segundo momento, el propósito fue estudiar las prácticas de cuidado de niños y niñas menores de 4 años realizadas por las familias en situación de pobreza, su distribución según sexo/género, y las estrategias desplegadas, especialmente por las mujeres, para resolver el cuidado infantil.

Es decir, podemos reconocer múltiples recursos institucionales articulados a diversas políticas públicas y redes de cuidados en el área de estudio. Analizamos especialmente en este trabajo, la dimensión educativa (nivel inicial) en tanto constituye uno de los servicios públicos de cuidado más importantes en niñoslas de la franja etaria de nuestro estudio.

En el siguiente apartado describimos y analizamos la oferta de cuidados, a partir del uso de cartografías y metodologías de geo referenciamiento (QGIS) incorporando al documento alguno de los mapas elaborados por el equipo para dar cuenta de la potencia de la metodología para el trabajo social.

\section{Caracterización del área bajo estudio}

La zona noroeste de la Ciudad de Paraná, capital de la Provincia de Entre Ríos, representa el 5,8\% de su población urbana (INDEC Censo 2010$)^{46}$ y constituye una de las zonas con mayor concentración de niñas y niños de hasta 4 años. La presencia de estos menores alcanza un décimo de sus habitantes (10,8\%), lo que supone un $43,2 \%$ más de niñas y niños que en el promedio de la ciudad.

46 El área es delimitada por la Fracción Censal 1 del Censo Nacional de Población, Hogares y Viviendas correspondiente a la ciudad de Paraná. Fuente: INDEC. 
Es una de las zonas con mayores niveles de pobreza y vulnerabilidad social con indicadores que duplican (e incluso triplican) los niveles medios de la ciudad INDEC - Censo 2010).

Con relación a los servicios de cuidados para niños y niñas menores de 4 años, tomamos como referencia el Censo de Población y la información de los jardines maternales municipales Dirección de Jardines Municipales, 2015), y realizamos un mapeo que da cuenta de la distribución geográfica de la oferta.

En el mapa correspondiente a la distribución de Jardines Municipales (Fig. 1), se muestra la matrícula por Jardín en relación con la densidad poblacional en las edades seleccionadas.

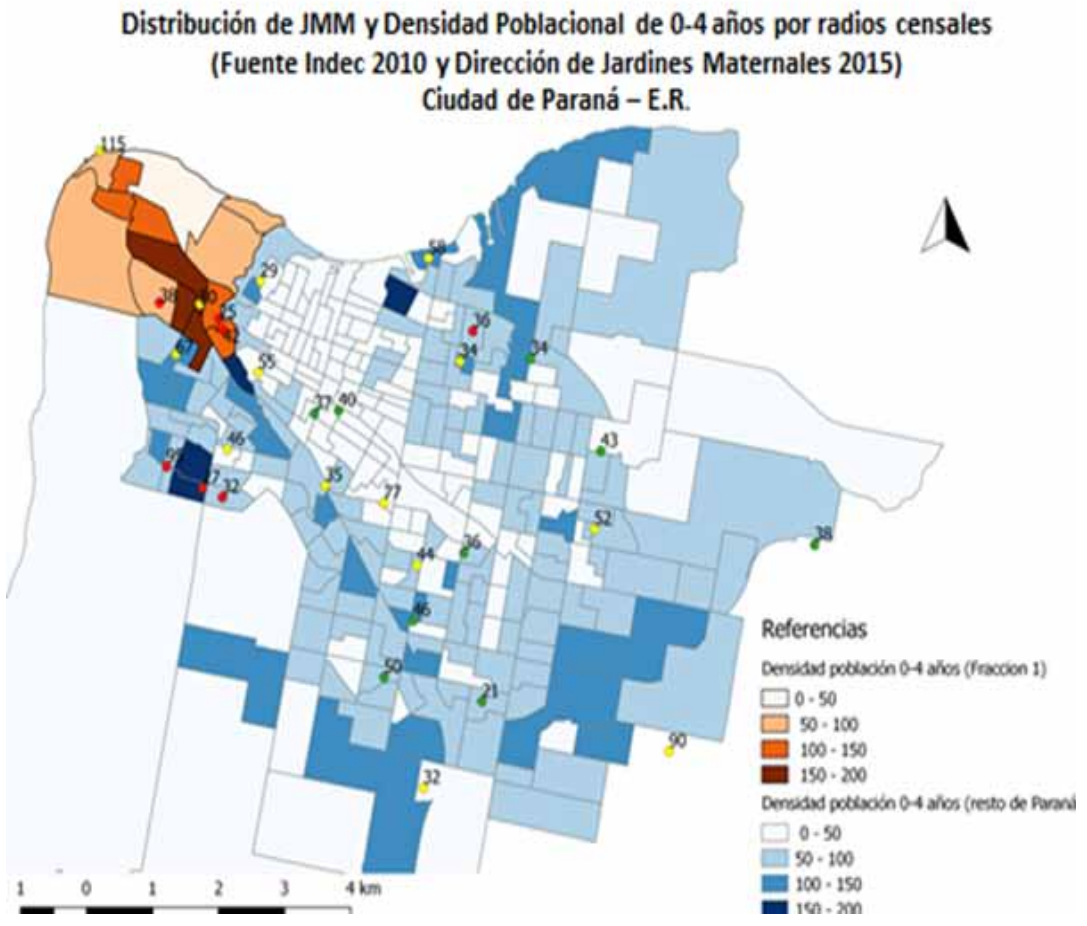

Fig. 1 - Fuente: Elaboración propia. 2018 
Así, es posible observar que en los "márgenes" de la ciudad, en particular en el área de referencia de esta investigación, se concentran los mayores valores de densidad poblacional de niños y niñas de 0-4 años. La oferta pública de espacios de cuidados no refleja criterios de concentración poblacional infantil y resulta insuficiente (déficit de cobertura y franja horaria limitada). Amplias zonas de la ciudad no cuentan con servicios gratuitos de cuidados.

Los jardines maternales municipales no se distribuyen con criterios de cobertura y accesibilidad en igualdad de condiciones para diferentes sectores Muy por el contrario, se observa que la política de servicios públicos de cuidado no llega a las zonas de mayor número de hogares pobres, ni donde se concentran los mayores valores de densidad poblacional de niños y niñas de 0-4 años.

En la cartografía se puede observar la relación entre cupos disponibles en jardines maternales municipales, y demanda real (expresada en las listas de espera) y la demanda potencial estimada en relación con la densidad poblacional de ese rango de edades.

En la zona delimitada por la investigación son 10 las instituciones educativas que atienden a niñas y niños hasta los 5 años. Cinco de ellas pertenecientes al Programa de Jardines Maternales de la Municipalidad, cuatro jardines de gestión pública provincial y uno de gestión privada. El déficit de la oferta de cuidados por parte del Estado es notable en la zona bajo estudio. Los registros del programa municipal cuentan con una la lista de espera de 86 niñas y niños, sobre una matrícula de 300 . Por su lado, los jardines privados y a cargo del estado provincial alcanzan una matrícula de 346 niñas y niños entre 3 y 5 años.

Si comparamos la matrícula total de 646 menores hasta 5 años (contabilizando Jardines municipales, privados y provinciales) con la población estimada de 1968 menores en ese tramo de edad, podemos observar que sólo el 32,8\% de ellos recibe regularmente algún tipo de cuidado fuera del ámbito familiar.

Otros aspectos importantes son el alcance y la disponibilidad horaria de estos servicios. Los cinco jardines municipales reciben a niñas y niños desde que se convierten deambuladores, quedando ex- 
cluidos los bebés. Tres de ellos cuentan con jornada simple, y dos con doble turno (Fig. 2).
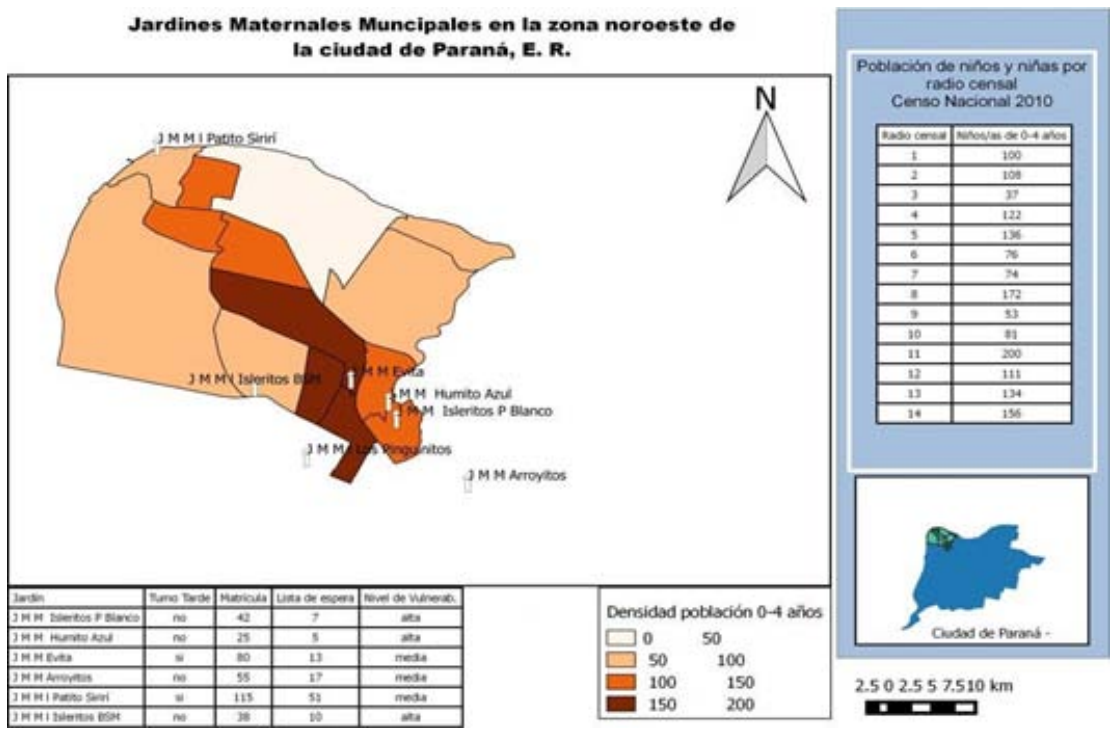

Fig. 2 - Fuente: Elaboración propia. 2018

Además de las instituciones educativas, se identifican en la zona de estudio la presencia de diversas organizaciones comunitarias que, en un sentido amplio, contribuyen con las prácticas de cuidados sobre todo en lo que respecta a recursos materiales (alimentación y vestimenta). Tal como plantea Zibechi (2013), estas organizaciones son diversas respecto de sus orígenes, modalidades, perspectivas (copas de leche, comedores comunitarios, organizaciones sociales diversas, grupos de diferentes iglesias, entre otras). Esta heterogeneidad también se aprecia en el grado de institucionalización, presupuestos e infraestructura, algunas cuentan con escasos recursos económicos y mínimo personal, otras cuentan con proyectos educativos robustos y con profesionales encargadas de llevar a cabo las tareas de cuidado. Coincidimos con Pautassi y Zibecchi (2013) en cuanto al planteo de que este tipo de estas organizaciones se encuentran en lugares donde 
residen familias de bajos recursos, donde el Estado ofrece poca o ninguna política de cuidados hacia la primera infancia.

En nuestro contexto de estudio esta oferta está constituida por diferentes ONGs, organizaciones comunitarias y voluntariado compuestas mayoritariamente por mujeres. Podemos afirmar que en tales espacios no se observa una perspectiva del cuidado como una cuestión social que trasciende al grupo familiar o doméstico.

\section{Conclusiones}

En este proyecto utilizamos la cartografía, entendida como un modo posible para graficar, visibilizar y señalar la distribución desigual de las ofertas de los servicios sociales públicos y privados en materia de cuidados. La intención de utilizar herramientas de geo referenciamiento se vincula con el objetivo de territorializar las políticas públicas y las organizaciones comunitarias vinculadas al cuidado, para construir un diagnóstico que dé cuenta de lo que el Estado y la comunidad "ofrece", a partir de las fuentes secundarias de datos y de las entrevistas a referentes de cada una de las organizaciones e instituciones del área de estudio. El objetivo es construir mapas que den cuenta de la desigualdad, que puedan mostrar la calidad de la oferta de los servicios públicos y políticas sociales y su peso en las condiciones de pobreza en los territorios referenciados.

La concentración de la pobreza en vinculación con escasa disponibilidad de servicios e infraestructura para los cuidados de niños/as y su distribución, es central en la conformación de las prácticas familiares y las estrategias para resolver el problema de los cuidados. Los patrones de desigualdad de los cuidados están vinculados entonces a desigualdades en términos sociales, económicos y de género.

Se advierte el papel de las responsabilidades familiares y de cuidado (no solo de niños y niñas sino de personas con discapacidad o de adultos y adultas mayores) en el acceso al trabajo remunerado y en la autonomía económica de las mujeres. A diferencia de mujeres de sectores económicos medios y altos, estas mujeres no pueden derivar este trabajo de cuidados en el mercado (aunque se identifican redes familiares y comunitarias en las que hay intercambio económico en 
lo que podríamos llamar un "mercado informal de cuidados"). La importancia de las familias y del trabajo no remunerado de las mujeres en la provisión del bienestar cotidiano y sus consecuencias no es visibilizado, por el contrario, reforzado por políticas públicas y algunas organizaciones comunitarias. La concentración de la oferta pública es insuficiente, por otro lado, no es accesible, su ubicación y distribución es desigual territorialmente. La mayor parte de la oferta de los jardines disminuye a medida de la edad de los niños es menor y que la pobreza es mayor. En una especie de efecto perverso el mercado avanza en proveer cuidados para los que pueden pagar cubriendo sobre todo edades más tempranas. Falta mucho andar aun para que los servicios de cuidado se configuren como derecho, el derecho a cuidar (o no) y el derecho a ser cuidado y cuidada.

\section{Referencias bibliográficas}

Addams, Jane (2013). Hull House: El valor de un centro social. Consejo General del Trabajo Social. Editorial. Paraninfo. España.

Addams, Jane (2014). El largo camino de la memoria de las mujeres. Zaragoza España, Prensas de la Universidad de Zaragoza.

Aguirre, Rosario (2007) “Trabajar y tener niños: insumos para repensar las responsabilidades familiares”. En: Gutiérrez, Ma. Alicia. Género, familias y trabajo: rupturas y continuidades. Desafíos para la investigación política. Buenos Aires, Argentina.

Arriagada, Irma (2005). Los límites del uso del tiempo: dificultades para las políticas de conciliación familia y trabajo". Reunión de expertos: Políticas hacia las familias protección e inclusión sociales. Buenos Aires, Argentina, CEPAL.

Batthyany, Karina (2006). Género y cuidados familiares. ¿Quién se hace cargo del cuidado y atención de los niños y adultos mayores? En: Fassler, Clara (coordinadora). Familias en cambio en un mundo en cambio, Trilce. Red Género y Familia, Montevideo.

Batthyany, Karina; Genta, Natalia y Scavino, Sol (2017). Análisis de género de las estrategias de cuidado infantil. En: Uruguay Cuadernos de Pesquisa, v.47, n.163, (pp. 292-319), jan./mar. 2017.

Bourdieu, Pierre (1997). Razones prácticas, sobre la teoría de la acción. Barcelona, España, Ed. Anagrama.

Bourdieu, Pierre (2007). El sentido práctico. Bs. As., Argentina, Siglo XXI Editores. 
Denzin, Norman y Lincoln, Yvonna (1994). Handbook of Qualitative Research, Thousand Oaks. California, Estados Unidos, Sage Publications Inc.

Esquivel, Valeria; Faur, Eleonor y Jelin, Elizabeth (2012). Las lógicas del cuidado infantil. Entre las familias, el estado y el mercado. Buenos Aires, IDES.

Guber, Roxana (2002) Trabajo Social y Trabajo de Campo. Reflexiones sobre un origen no registrado. En: La Investigación en Trabajo social. Publicación Post Jornadas. Facultad de Trabajo Social. UNER.

Jelin, Elizabeth (1980). Trabajo y Familia en el ciclo de vida femenino: El caso de los sectores populares de Buenos Aires. Buenos Aires, Argentina, Estudios CEDES.

Matus, Teresa (2017). Materiales de una crítica: relatos, mapas y datos. En: Warner, Ma. alejandra y Rozas Pagaza Margarita (Comp.), "Igualdad y desigualdad social en América Latina: generando debates en Trabajo Social en relación con otras ciencias del campo social": III Foro Latinoamericano de trabajo social. Ciudad Autónoma de Bs. As., Editorial Espacio.

Pautassi, Laura y Zibecchi, Carla (2013). Las fronteras del cuidado. Buenos Aires, Argentina, Biblos.

Perez Orozco, Amaia (2009). Miradas globales a la organización social de los cuidados en tiempos de crisis. ¿Qué retos políticos debemos afrontar? Serie Género, Migración y Desarrollo, Naciones Unidas, República DominicanTaylor, Steven y Bogdan, Robert, (1986). Introducción a los métodos cualitativos de investigación, Buenos Aires, Paidós.

Travi, Bibiana (2015). Jane Addams, pionera de la sociología y del Trabajo Social: la memoria y la visibilización de la violencia contra las mujeres. En: Revista "Debate Público. Reflexión de Trabajo Social” - Recuperando historia, Año 5 - Nro. 9. UBA. Buenos Aires.

Vasilachis Inés (1993). Métodos cualitativos I. Los problemas teórico-epistemológicos, Buenos Aires, CEAL.

Recepción: 27/02/2020

Aceptación: 12/08/2020 